\title{
Beauvericin-induced cell apoptosis through the mitogen-activated protein kinase pathway in human nonsmall cell lung cancer A549 cells
}

\author{
Chien-Lin Lu', Hen-I Lin ${ }^{1,2}$, Bing-Fang Chen ${ }^{1}$ and Guey-Mei Jow ${ }^{1}$ \\ ${ }^{1}$ School of Medicine, Fu Jen Catholic University, New Taipei City, Taiwan \\ ${ }^{2}$ Department of Internal Medicine, Catholic Cardianal Tien Hospital, New Taipei City, Taiwan
}

(Received December 31, 2015; Accepted April 18, 2016)

\begin{abstract}
Beauvericin (BEA) is a cyclic hexadepsipeptide that derives from Codyceps cicadae. Our previous study results indicated that the cytotoxic effects of BEA on human A549 lung cancer cells BEA occur through an apoptotic pathway, which involves the up-regulation of cytochrome c release from mitochondria, upregulation of caspase 3 activity, and cellular and morphological changes. In this study, we identified that the mitogen-activated protein kinase (MAPK) inhibitor U0126 inhibits the cytotoxic effects of BEA on A549 cells. After exposing human A549 cells to $10 \mu \mathrm{M}$ BEA, we observed a significant and dose-dependent increase in the percentage of hypoploid (sub-G1) phase cells in the A549 population. Following the pretreatment of the A549 cells with $25 \mu \mathrm{M} \mathrm{U} 0126$, the distribution of A549 cells in the sub-G1 phase decreased significantly. The BEA treatment resulted in a significant increase apoptosis in A549 cells by in situ terminal deoxynucleotidyl transferase dUTP nick end labeling (TUNEL) assay. Moreover, the MEK1/2 (mitogen-activated protein kinase kinase)-ERK42/44 (extracellular signal-regulated kinases)-90RSK (ribosomal s6 kinase) signaling pathway was activated in BEA-induced apoptotic A549 cells. Furthermore, treatment with MEK1/2 inhibitor U0126 was capable to attenuate the BEA induced typical apoptotic morphological change, apoptotic cells, and MEK1/2-ERK42/44-90RSK signaling pathway. These results suggested that MEK1/2-ERK42/44-90RSK signaling pathway may play a important role in BEA-induced apoptosis in human NSCLC A549 cancer cells.
\end{abstract}

Key words: Beauvericin, MAPK, Apoptosis

\section{INTRODUCTION}

In Taiwan, lung carcinoma is the leading cause of cancer-related mortality and more than $75 \%$ of lung cancers are nonsmall cell lung cancers (NSCLC). Chemotherapy is the standard treatment for advanced (stage IIIB and IV) NSCLC patients and provides a relatively high performance status $(0,1$, and 2$)$. Although chemotherapy can substantially improve the symptoms and quality of life of patients with advanced NSCLC, only a modest increase in survival rate is typically achieved (Bonomi et al., 2000; Cardenal et al., 1999; Sandler et al., 2000; ten Bokkel Huinink et al., 1999). Therefore, novel approaches are required for the treatment of advanced NSCLC to increase patient survival or improve palliative care. The development of chemotherapeutic or chemopreventive agents with maximal antitumor activity, and minimal unwanted toxic side effects, is thus crucial.

The mitogen-activated protein kinases (MAPKs) are a family of Ser/Thr protein kinases that participate in extracellular signal transmission in the nucleus (Schaeffer and Weber, 1999). Three subfamilies of MAPKs have been identified: extracellular signal-regulated protein kinase (ERK), c-Jun NH2-terminal kinase (JNK), also known as stress-activated protein kinase (SAPK), and p38 MAPK. These MAPKs require phosphorylation on one threonine (Thr) and one tyrosine (Tyr) residue for activation to occur (Robinson and Cobb, 1997). SAPK/JNK and p38 MAPK are considered to be associated with the induction of apoptosis (Chuang et al., 2000; Kunz et al., 2001; Stadheim et al., 2000). Studies have reported that SAPK/ JNK and p38 MAPK act upstream of mitochondrial malfunction and caspase activation in several cell types. For example, the stimulation of p38 MAPK is an early regula-

Correspondence: Guey-Mei Jow (E-mail: 039666@mail.fju.edu.tw) 
tory event in the cadmium-induced apoptotic pathway in human promonocytic cells (Cheng et al., 2001; Galán et al., 2000). Activated MAPKs can phosphorylate several transcriptional factors, such as E1k-1, ATF-2, and c-jun. They also regulate the expression of the c-fos and c-jun genes (Karin, 1995).

Beauvericin (BEA), a hexadepsipeptide mycotoxin, has been recognized as an important toxic compound by the insect pathogen Beauveria bassiana (Hamill et al., 1969). It has been shown that BEA displays broad spectrum antiproliferative activity against different human cancer cells lines by activating calcium-sensitive cell apoptotic pathways (Jow et al., 2004; Ojcius et al., 1991; Calò et al., 2004). Furthermore, our previous study results indicated that beauvericin (BEA)-induced NSCLCA549 cytotoxicity occurs through an apoptotic pathway, which involves the upregulation of cytochrome c release from mitochondria, upregulation of caspase 3 activity, and cellular and morphological changes (Lin et al., 2005). In this study, we investigated the induction of apoptosis in NSCLC A549 cells by BEA through MAPKs signal transduction pathway.

\section{MATERIALS AND METHODS}

\section{Materials and cell culture}

The A549 cells were cultured in Dulbecco's Modified Eagle Medium (DMEM, Hyclone, UT, USA) supplemented with $10 \%$ heat-inactivated fetal bovine serum (Hyclone Lab.), $100 \mu \mathrm{g} / \mathrm{mL}$ penicillin, $100 \mu \mathrm{g} / \mathrm{mL}$ streptomycin, and $100 \mu \mathrm{g} / \mathrm{mL}$ amphotericin B (Hyclone Lab.). The cells were grown in a humidified incubator at $37^{\circ} \mathrm{C}$ in an atmosphere containing $5 \%$ carbon dioxide and $95 \%$ air. In each experiment, $3 \times 10^{5}$ cells were seeded in wells in a 24 -well plate containing $1 \mathrm{~mL}$ of fresh medium, and then incubated with or without chemical treatment for an indicated duration. In the toxicity experiments, cells were treated with BEA during the exponential phase of cell growth.

BEA was obtained from Dr. Cheng-Jen Chou (National Research Institute of Chinese Medicine) and dissolved in pure grade dimethyl sulfoxide (DMSO). Human NSCLC A549 cells were obtained from ATCC. Monoclonal antibodies of MAPKs, and antirabbit IgGs were purchased from Cell Signaling, Inc. (Danvers, MA, USA). All chemicals were of the highest available purity and grade.

\section{Cytotoxicity assay}

The cytotoxic effects of BEA on the human NSCLC A549 cells were determined using the 3-(4,5-dimethylthiazol-2-yl)-2,5-diphenyl tetrazolium bromide (MTT) method (Mosmann, 1983). MTT was dissolved in phosphate-buffered saline (PBS) and filtered. In brief, $100 \mu \mathrm{L}$ of MTT per $1 \mathrm{~mL}$ of the medium was added to each well. After reaction with the MTT, the medium was replaced with $1 \mathrm{~mL}$ of pure DMSO for color development. The absorbance density values of the wells were then measured using an enzyme-linked immunosorbent assay (ELISA) reader at $570 \mathrm{~nm}$ to determine cell viability, with the DMEM referenced as a blank.

\section{Flow cytometric analysis of apoptosis}

The human NSCLC A549 cells were cultured with 0, 1,5 , or $10 \mu \mathrm{M} / \mathrm{L}$ BEA with or without U0126 for $24 \mathrm{hr}$, collected and rinsed with PBS, and suspended in $75 \%$ ethanol at $-20^{\circ} \mathrm{C}$ overnight. Fixed cells were centrifuged at 1,200 $\mathrm{g}$ and washed twice with PBS. To detect DNA content, cells were contained in the dark with $50 \mathrm{mg} / \mathrm{L}$ propidium iodide $(\mathrm{PI})$ and $0.1 \%$ ribonuclease $\mathrm{A}$ in $400 \mu \mathrm{L}$ of $\mathrm{PBS}$ at $25^{\circ} \mathrm{C}$ for $30 \mathrm{~min}$. Stained cells were analyzed using a FACSort flow cytometer (Becton Dickinson, Franklin Lakes, NJ, USA). The percentage of apoptotic cells was determined using CellQuest software. By analyzing the DNA histograms of cells, the percentages of the cells in various cell cycle phases could be evaluated. Cells with DNA content that did not progress beyond the G1 phase (sub-G1) were considered apoptotic cells.

\section{Morphological analysis and TUNEL assay}

In situ detection of apoptotic cells were carried out using TUNEL apoptosis detection kits. The in situ TUNEL assay identifies apoptosis cells using terminal deoxynucleotidyl transferase (TdT) to transfer biotindUTP to the free $30 \mathrm{OH}$-ends of cleaved DNA. The biotinlabeled cleavage sites were then visualized by reaction with fluorescein conjugated with avidin (avidin-fluorescein isothiocyanate). Human NSCLC A549 cells were cultured on chamber slides for $24 \mathrm{hr}$ and then treated with BEA with or without U0126 for overnight. After incubation, the cell morphology was imaged by inverted phase contrast microscopy (Olympus, Tokyo, Japan). Then, the cells were washed with PBS twice and fixed with ethanol/ acetic acid for TUNEL assay. Then, the cells were stained with TUNEL staining according to the protocols provided by the suppliers. The morphology of treated cells was examined using an Olympus fluorescence microscope and 500 stained cells from each treatment group were counted.

\section{Western blot analysis}

Cell lysates were prepared by extracting proteins using a lysis buffer [20 mM/L Tris-HCl, pH7.5 $150 \mathrm{mM} / \mathrm{L}$ 
Role of MAPK in beauvericin-induced cell apoptosis

$\mathrm{NaCl}, 1 \mathrm{mM} / \mathrm{L}$ ethylenediaminetetraacetic acid (EDTA), $1 \mathrm{mM} / \mathrm{L}$ ethylene glycol tetraacetic acid (EGTA), 1\% Triton, and $1 \mathrm{mM} / \mathrm{L}$ phenylmethylsulfonyl fluoride (PMSF)], supplemented with protease inhibitors. The proteins were separated using sodium dodecyl sulfate polyacrylamide gel electrophoresis (SDS-PAGE) and then transferred onto a polyvinylidene fluoride (PVDF) membrane. The membrane was blocked with $5 \%$ nonfat dry milk in Trisbuffered saline and then incubated with the primary antibodies for $1 \mathrm{hr}$ at room temperature. The blots were then developed with a peroxidase-conjugated secondary antibody, and the proteins visualized using enhanced chemiluminescence procedures (Amersham, Arlington Heights, IL, USA) according to the manufacturer's instructions.

\section{Statistical analysis}

Data are presented as mean \pm S.D. for the indicated number of experiments. All statistical analyses were performed using a one-way analysis of variance (ANOVA) followed by a $t$ test, with ${ }^{*} P<0.01$ and ${ }^{* *} P<0.001$ considered significant.

\section{RESULTS}

In this study, we evaluated the effects of BEA and MAPK kinase inhibitors on human NSCLC A549 cell viability and MAPK expression. We pretreated the cells with MAPK kinase inhibitors for $1 \mathrm{hr}$ prior to exposure to $10 \mu \mathrm{M}$ BEA (24 hr). We observed that the MEK inhibitor U0126 (MEK selective inhibitor) inhibited BEA-induced cytotoxicity (Fig. 1). In contrast, the LY294002 (PI3K inhibitor) did not affect BEA-induced cytotoxicity, whereas SB202190 (p38 inhibitor) partially inhibited BEA-induced cytotoxicity. SB202190, LY294002, and U0126 are all inhibitors of MAPK signaling. However, the affinity of U0126 to MEK is substantially higher than that of SB202190, and LY294002. Therefore, we used U0126 for MEK inhibition in subsequent experiments.

Mitogen-induced ERK activation is essential for transient G1-phase arrest (Roovers and Assoian, 2000). In this study, we used flow cytometric analysis to determine the role of MEK in the cell cycle phase profiles affected by BEA. As shown in Fig. 2, BEA treatment increased the sub-G1 phase proportion of the A549 cells in a dosedependent manner. Our results also indicated that exposure of the A549 cells to $10 \mu \mathrm{M}$ BEA for $24 \mathrm{hr}$ markedly reduced the $\mathrm{G} 0 / \mathrm{G} 1$ phase and $\mathrm{G} 2 / \mathrm{M}$ phase proportions, and marginally increased the $\mathrm{S}$ phase cell proportion. These findings suggested that cells might have accumulated during the S phase, and were not arrested in the G0/ G1 or G2/M phases. U0126 pretreatment increased G0/ G1 phase, and reduced sub-G1 phase, S-phase, and G2/M phase, A549 cell proportions after BEA treatment (Figs. $2 \mathrm{~A}, 2 \mathrm{~B}$, and 3 ). These results suggested that U0126 might have increased cell accumulation in the late G1 phase in the presence of BEA.

To determine whether ERK1/2 activation is involved in the signal transduction pathway involved in BEA-induced cytotoxicity, several identification techniques were carried out in this study. Morphological examination revealed that $10 \mu \mathrm{M}$ BEA caused A549 cells to round up and lose the cell contact (Fig. 4d), and further detach from the

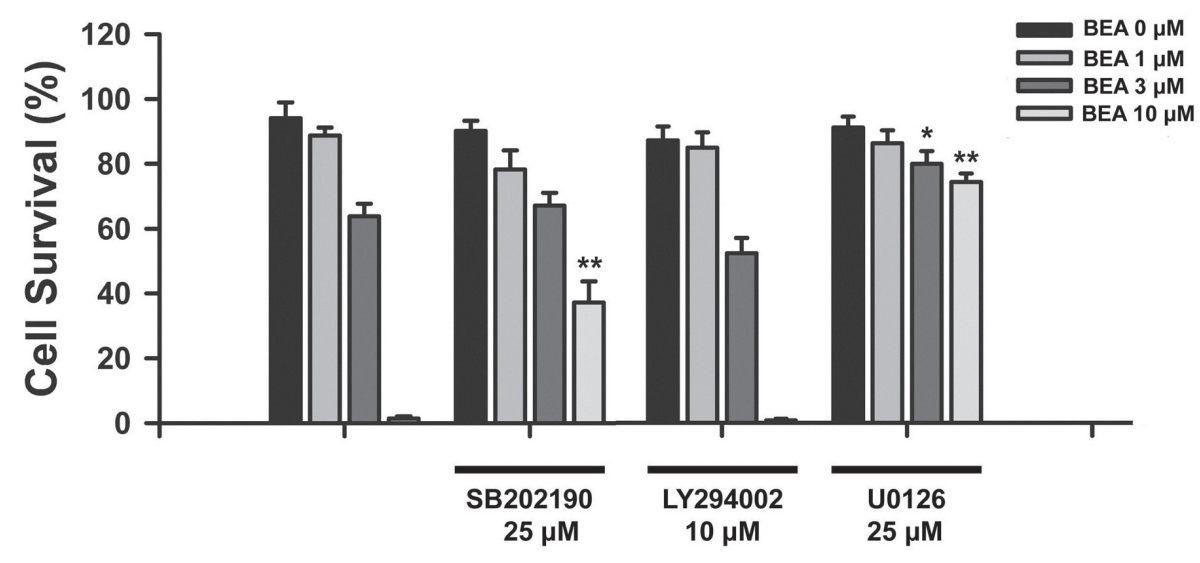

Fig. 1. Effect of several MAPK inhibitors on beauvericin-induced cytotoxicity in human A549 cells. Cells were incubated in either the absence (basal and control) or presence of the indicated inhibitors for $2 \mathrm{hr}$. Subsequently, either $1 \mu \mathrm{M}, 3 \mu \mathrm{M}, 10 \mu \mathrm{M}$ of $\mathrm{w}$ beauvericin were added to the cells for $24 \mathrm{hr}$. Cytotoxicity effect was detected by the MTT assay method as described in Materials and Methods section. Cytotoxicity is expressed as a percentage of reduction in mitochondrial MTT activity and the data are expressed as mean \pm S.D. of $4-5$ determinations, ${ }^{*}: p<0.01$ and $^{* *}: \mathrm{p}<0.001$. 


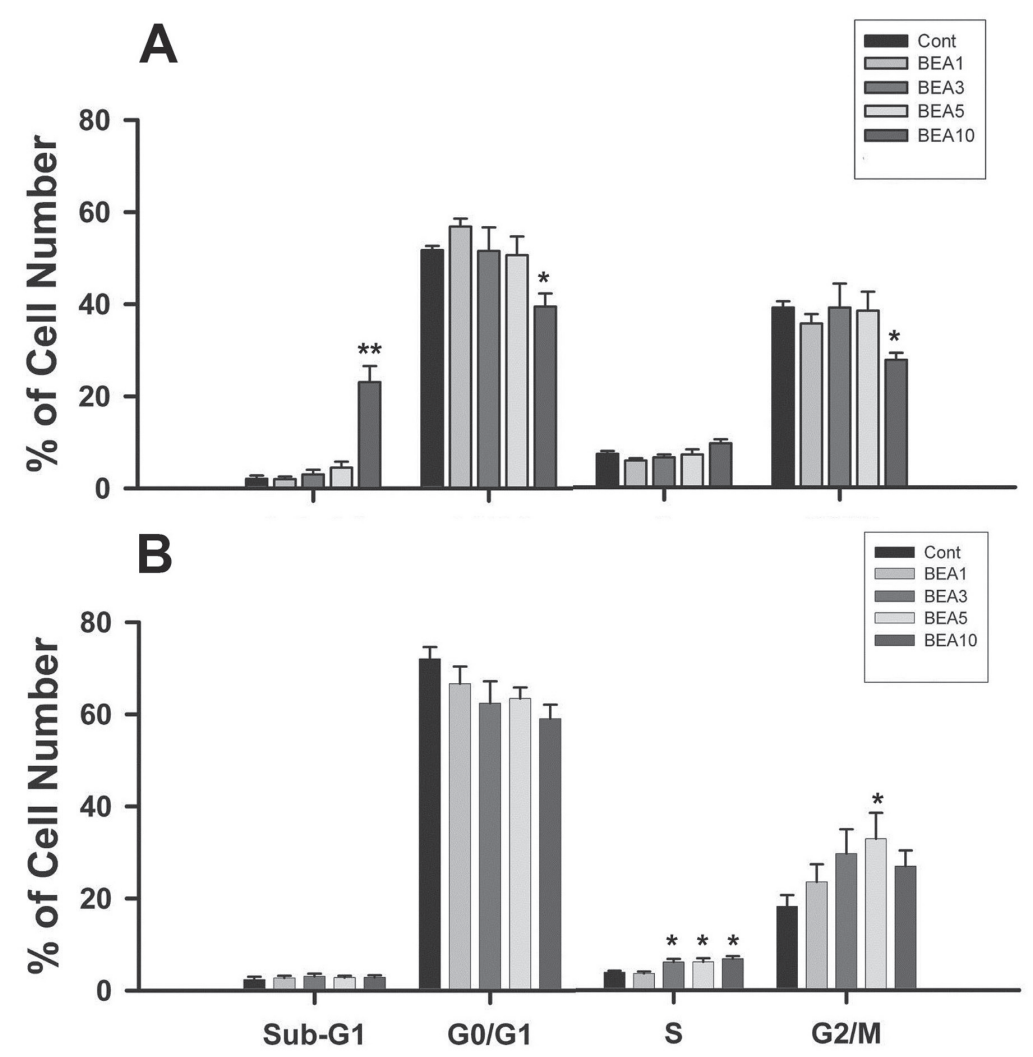

Fig. 2. Effects of MEK inhibitors U0126 on beauvericin-induced cell cycle change in human NSCLC A549 cells. Human A549 cells were pretreated without (A) or with (B) MEK inhibitor U0126 (25 $\mu \mathrm{M})$ for 30 min and then were treated with the indicated concentration of beauvericin or and an equal volume of vehicle DMSO (control) and examined after $24 \mathrm{hr}$ for DNA degradation ( $\%$ of hypoploid cells) characteristic of apoptosis using hypotonic PI staining. The hypoploid cells have less cellular DNA content than cell typically found in the G0/G1, S or G2/M phases of the cell cycle, which causes them to retain less PI, a fluorescent DNA stain. The decreased retention of PI by the hypoploid cells shifts their fluorescence intensity leftward on the linear $\mathrm{X}$-axis of the representative histograms.

culture plates. Pretreatment with MEK inhibitor U0126 $(25 \mu \mathrm{M})$ attenuated these morphological changes after co-incubated with BEA for overnight (Fig. 4h). The hallmarks of apoptosis are nuclear chromatin condensation and fragmentation of DNA strand using in situ TUNELreactions. As demonstrated in Fig 5, the control cells showed negative staining (upper left panel), while BEA $(10 \mu \mathrm{M})$ significantly induced the cell apoptosis with strong fluorescence staining (upper right panel). However, the TUNEL-positive cells significantly diminished by pretreatment with U0126 (lower right panel). These results suggested that the ERK pathway might involve in BEA-induced cytotoxicity.

To investigate the mechanism by which BEA induces A549 cytotoxicity through the activation of cell signaling cascades involving MAPKs, the kinetics of ERK42/44, MEK1/2, and 90RSK phosphorylation was analyzed by
Western blot analysis. The results clearly showed that phosphorylated ERK42/44 (p-ERK42/44) expression was activated by BEA treatment at $3 \mu \mathrm{M}$ and $10 \mu \mathrm{M}$ concentration (Fig 6, left panel). Meanwhile, BEA also induced MEK1/2 (the upstream of ERK42/44) and 90RSK protein (activated by MAPK-ERK pathway) activation (Fig. 6, left panel). However, the MEK inhibitor U0126 was significantly downregulated BEA-induced MEK1/2ERK42/44-90RSK activation (Fig. 6 right panel). U0126 prevented the BEA induces MEK-ERK-90RSK activation that followed a very congruent pattern indicating a direct correlation between them. These data indicate that BEA induces cell apoptosis may through the MEK1/2ERK42/44-90RSK signaling pathway in A549 cells. 
Role of MAPK in beauvericin-induced cell apoptosis
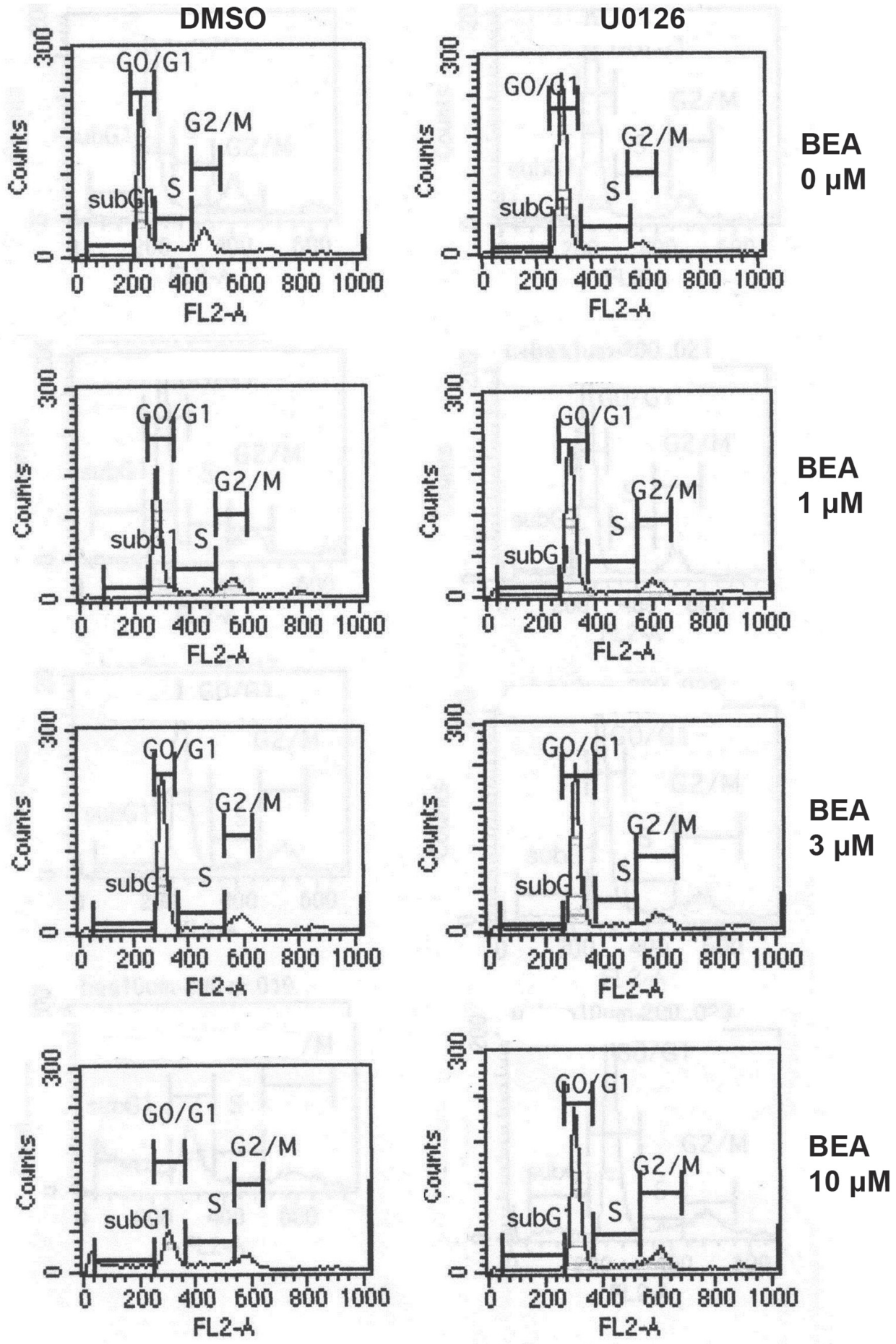

Fig. 3. Evaluation of the effects of U0126 on BEA-induced apoptosis in human NSCLC A549 cells. Human A549 cells were pretreated without (left panels) and with MEK inhibitor U0126 $(25 \mu \mathrm{M})$ for $30 \mathrm{~min}$ and then were treated with the indicated concentration of beauvericin or and an equal volume of vehicle DMSO (control) and examined after $24 \mathrm{hr}$ for DNA degradation (\% of hypoploid cells) characteristic of apoptosis using hypotonic PI staining. The hypoploid cells have less cellular DNA content than cell typically found in the G0/G1, S or G2/M phases of the cell cycle, which causes them to retain less PI, a fluorescent DNA stain. The decreased retention of PI by the hypoploid cells shifts their fluorescence intensity leftward on the linear $\mathrm{X}$-axis of the representative histograms. 


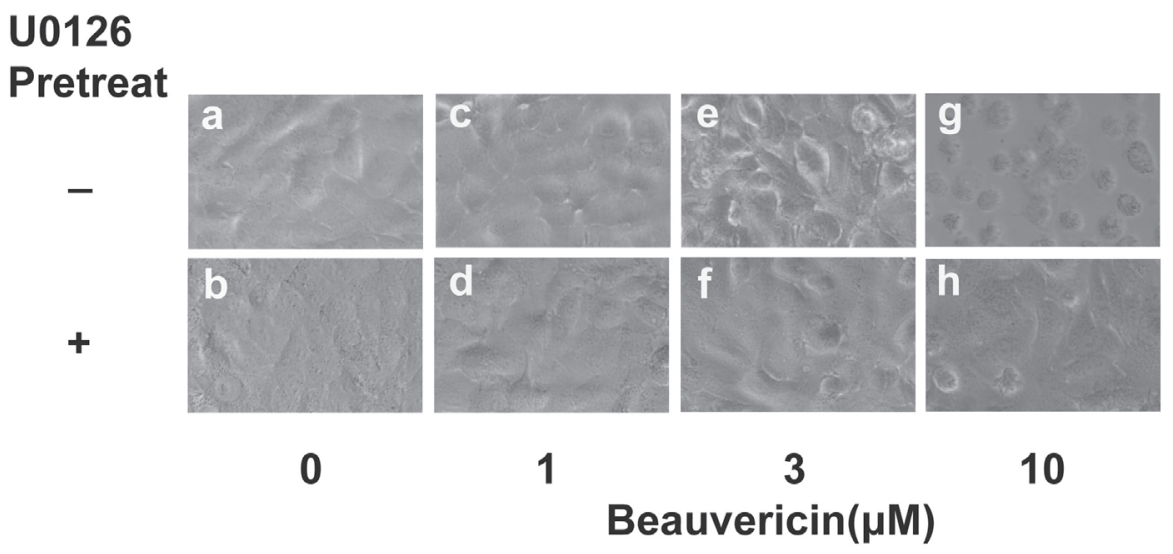

Fig. 4. Effect of U0126 on cell morphological change in BEA-treated human NSCLC A549 cells by phase contrast microscopy. Human A549 cells were pretreated without (upper) or with (lower) MEK inhibitor U0126 $(25 \mu \mathrm{M})$ for $1 \mathrm{hr}$ and then were treated with the indicated concentration of beauvericin and an equal volume of vehicle DMSO (control) for overnight. Then, the cell morphological change was detected by Olympus inverted phase contrast microscopy as described in Materials and Methods section.

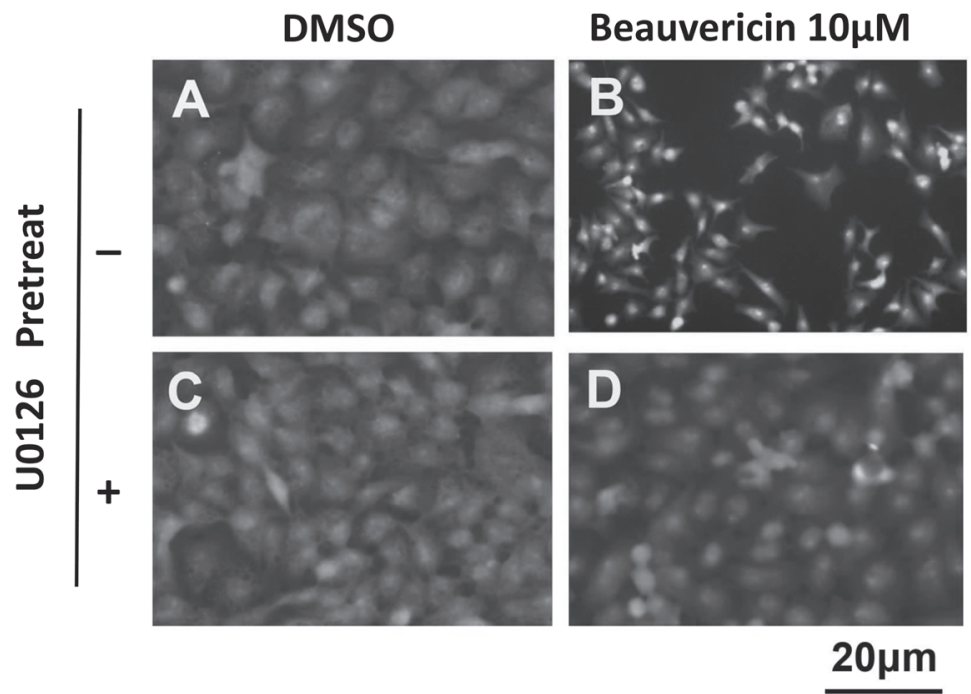

Fig. 5. Effect of U0126 on the BEA-induced apoptosis in human NSCLC A549 cells by in situ TUNEL assay. Human NSCLC cells were cultured onto chamber slide for $24 \mathrm{hr}$ for attachment. Human A549 cells were pretreated without (upper) or with (lower) MEK inhibitor U0126 (25 $\mu \mathrm{M})$ for $1 \mathrm{hr}$ and then were treated with $10 \mu \mathrm{M}$ BEA and an equal volume of vehicle DMSO (control) for overnight. The numbers of apoptotic cells were examined by Olympus fluorescence microscope as described in Materials and Methods section.

\section{DISCUSSION}

Apoptosis is a complex but regulated mechanism that enables an organism to eliminate defective and unwanted cells through an inflammatory response. However, the dysregulation of apoptosis can trigger cancer, autoim- mune disease, neurocognitive disorders, and cardiovascular disease. Various signals can trigger apoptosis, including Fas ligand, tumor necrosis factor, growth factor withdrawal, viral or bacterial infection, oncogenes, irradiation, ceramide, and chemotherapeutic drugs (Evan et al., 1995). Several studies have confirmed the close asso- 


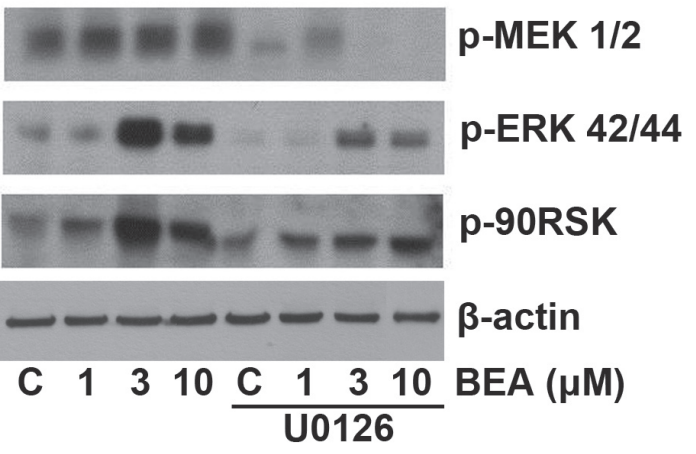

Fig. 6. The effect of U0126 on the BEA-induced MAPK kinase protein expression in NSCLC A549 cells. Human NSCLC A549 cells were treated without or with MEK inhibitor U0126 $(25 \mu \mathrm{M})$ for $2 \mathrm{hr}$ and then treated with BEA as indicated concentration for $24 \mathrm{hr}$. After the treatment, cell lysate were extracted, and then the levels of p-MEK1/2, p-ERK42/44, and p-RSK 90 proteins were analyzed by Western blot analysis.

ciations between the cell cycle and apoptotic responses, and evidence has demonstrated that apoptosis and the cell cycle share common molecular pathways (Lundberg and Weinberg, 1999). In a normal cell cycle, cells exit the quiescent state and progress from the G0 phase into the $\mathrm{G} 1$ phase. The $\mathrm{S}$ phase occurs subsequent to the $\mathrm{M}$ phase and the $\mathrm{M}$ phase is initiated after the completion of the S phase. The G1 and G2 phases separate the S and $\mathrm{M}$ phases. Several signaling checkpoints ensure that the cell cycle selectively activates pathways that lead to programmed cell death if cellular damage cannot be repaired (Pietenpol and Stewart, 2002). Tumor suppressor genes, such as $\mathrm{p} 53$ and $\mathrm{pRb}$, the dominant oncogene $\mathrm{c}-\mathrm{Myc}$, and several cyclin-dependent kinases (Cdks), all function to sensitize cells in preparation for apoptosis (Pucci et al., 2000). The role of $\mathrm{p} 53$ is essential because it induces G1 arrest by upregulating p21 expression, G2 arrest if the mitotic spindle is damaged, and $\mathrm{S}$ phase arrest if the substrates required for DNA synthesis are depleted (Taylor and Stark, 2000).

The MAPKs are a family of kinases that transduce signals from the cell membrane to the nucleus in response to various insults. The MAPKs consist of serine or threonine kinases that phosphorylate substrates that regulate gene expression, mitosis, proliferation, motility, metabolism, and programmed cell death (Chang and Karin, 2001). ERK1 and ERK2 are well characterized MAPKs that are activated in response to growth stimuli. JNKs and p38MAPK are simultaneously activated in response to vari- ous cellular and environmental stresses, such as changes in osmolarity or metabolism, DNA damage, heat shock, ischemia, inflammatory cytokines, or oxidative stress (Wada and Penninger, 2004). The ERK signaling cascade has been well described. Following activation, ERK translocates to the nucleus and phosphorylates various substrates, which exert proliferative or antiproliferative effects through downstream transcription factor targets, including MEF2, NF- $k$ B, Ets-1, RSK, and c-Myc. These factors then regulate the expression of numerous genes, such as Cdks, cyclins, and Bcl-2, which are involved in cell cycle progression and antiapoptotic functions (Chang et al., 2003).

Previous studies had demonstrated that BEA is a potent cytotoxic compound capable of inducing apoptosis in human acute lymphoblastic leukemia cells (Jow et al., 2004) and human NSCLC A549 cells (Lin et al., 2005). We reported BEA had potent cytotoxicity effect in human NSCLC A549 cells in concentration-dependent manner but didn't cause any cell loss in $\mathrm{HaCa}$ cells in even $30 \mu \mathrm{M}$ (IC50 at $24 \mathrm{hr}$ exposure was $4.5 \mu \mathrm{M}$ ). Namely, BEA had the specificity of the cytotoxicity to cancer cell instead of normal cell. By upregulating Bax, Bak and downregulating $\mathrm{p}-\mathrm{Bcl}-2$ expression, BEA significantly reduced mitochondrial membrane potential and activates caspase- 3 apoptotic cascade to exert it apoptotic death. Furthermore, $24 \mathrm{hr}$ treatment with BEA exhibited characteristic morphological features of apoptosis, such as membrane shrinkage and chromosomal condensation in fluorescence microscopy.

To identify the mechanism underlying the inhibition of A549 proliferation through the ERK MAPK signaling axis, we pretreated cells with a potent inhibitor of MAPKs prior to BEA treatment. We evaluated the apoptotic effects of BEA according to the percentages of hypoploid sub-G1 cells in response to various BEA concentrations. We observed a marked increase in the sub-G1 population following exposure to $10 \mu \mathrm{M}$ BEA. However, U0126 pretreatment inhibited this effect. Generally, cytotoxicity disrupts cell cycle progression through antiproliferative effects, and is followed by the accumulation of cells in one or more phases of the cell cycle. After exposing A549 cells to $10 \mu \mathrm{M}$ BEA, we observed accumulation in the $\mathrm{S}$ phase, and reduced cell proportions in the corresponding G0/G1 and G2/M phases. This trend strongly suggested the impairment of mitosis because of arrest in the S phase. Cell cycle disruption could partially be explained by a checkpoint response from DNA damage or other mechanisms that require further investigation. Our observations on cell cycle behavior after exposure to BEA support previous results on the cytotoxic effects of 
dictamnine on A549 cells (An et al., 2013).

To further explore the association of ERK activation/ phosphorylation in BEA induced apoptotic cell death, morphological studies by phase contrast microscopy and in situ TUNEL assay disclosed BEA can obviously induced NSCLC A549 apoptotic change in concentrationdependent manner. However, these findings were inhibited by pretreatment with MEK inhibitor, U0126. The activation of RSK requires ordered phosphorylation events mediated by MEK/1/2-ERK42/44, phosphoinositide-dependent protein kinase 1 (PDK1), and RSK autophosphorylation. Studies have emphasized the role of RSKs in nuclear signaling, cell cycle progression, cell proliferation, cell growth, protein synthesis, and cell survival (Cargnello and Roux, 2011). Our results strongly suggest that BEA-induced cytotoxicity to NSCLC A549 cells involves the MEK1/2/-ERK42/44 pathway with 90RSK a downstream phosphorylated target.

In conclusion, BEA exerts anticancer effects by inducing A549 cell cycle arrest in the $\mathrm{S}$ phase and apoptosis though the MEK1/2/ERK42/44/90RSK signaling pathway. Our results also confirm the cytotoxic effects of BEA on the A549 cell line and support the potential use of BEA in future NSCLC treatments.

\section{ACKNOWLEDGMENTS}

This work was supported by grants NSC94-2320-B030-004 from the National Science Council and grants CTH-94-1-2B04 from the Catholic Cardinal Tien Hospital. We would like to thank Dr. C.J. Chou for his generosity to provide the drugs. We would also like to thank Ms. C.F. Yang for her excellent technical assistance.

Conflict of interest---- The authors declare that there is no conflict of interest.

\section{REFERENCES}

An, F.F., Liu, Y.C., Zhang, W.W. and Liang, L. (2013): Dihydroartemisinine enhances dictamnine-induced apoptosis via a caspase dependent pathway in human lung adenocarcinoma A549 cells. Asian Pac. J. Cancer Prev., 14, 5895-5900.

Bonomi, P., Kim, K., Fairclough, D., Cella, D., Kugler, J., Rowinsky, E., Jiroutek, M. and Johnson, D. (2000): Comparison of survival and quality of life in advanced non-small-cell lung cancer patients treated with two dose levels of paclitaxel combined with cisplatin versus etoposide with cisplatin: results of an Eastern Cooperative Oncology Group trial. J. Clin. Oncol., 18, 623-631.

Calò, L., Fornelli, F., Ramires, R., Nenna, S., Tursi, A., Caiaffa, M.F. and Macchia, L. (2004): Cytotoxic effects of the mycotoxin beauvericin to human cell lines of myeloid origin. Pharmacol. Res., 49, 73-77.
Cardenal, F., López-Cabrerizo, M.P., Antón, A., Alberola, V., Massuti, B., Carrato, A., Barneto, I., Lomas, M., García, M., Lianes, P., Montalar, J., Vadell, C., González-Larriba, J.L., Nguyen, B., Artal, A. and Rosell, R. (1999): Randomized phase III study of gemcitabine-cisplatin versus etoposide-cisplatin in the treatment of locally advanced or metastatic non-small-cell lung cancer. J. Clin. Oncol., 17, 12-18.

Cargnello, M. and Roux, P.P. (2011): Activation and function of the MAPKs and their substrates, the MAPK-activated protein kinases. Microbiol. Mol. Biol. Rev., 75, 50-83.

Chang, F., Steelman, L.S., Lee, J.T., Shelton, J.G., Navolanic, P.M., Blalock, W.L., Franklin, R.A. and McCubrey, J.A. (2003): Signal transduction mediated by the Ras/Raf/MEK/ERK pathway from cytokine receptors to transcription factors: potential targeting for therapeutic intervention. Leukemia, 17, 1263-1293.

Chang, L. and Karin, M. (2001): Mammalian MAP kinase signalling cascades. Nature, 410, 37-40.

Cheng, A., Chan, S.L., Mihavet, O., Wang, S. and Mattson, M.P. (2001): p38 MAP kinase mediates nitric oxide-induced apoptosis of neural progenitor cells. J. Biol. Chem., 276, 43320-43327.

Chuang, S.M., Wang, I.C. and Yang, J.L. (2000): Roles of JNK, p38 and ERK mitogen-activated protein kinases in the growth inhibition and apoptosis induced by cadmium. Carcinogenesis, 21, 1423-1432.

Evan, G.I., Brown, L., Whyte, M. and Harrington, E. (1995): Apoptosis and the cell cycle. Curr. Opin. Cell Biol., 7, 825-834.

Galán, A., García-Bermejo, M.L., Troyano, A., Vilaboa, N.E., de Blas, E., Kazanietz, M.G. and Aller, P. (2000): Stimulation of p38 mitogen-activated protein kinase is an early regulatory event for the cadmium-induced apoptosis in human promonocytic cells. J. Biol. Chem., 275, 11418-11424.

Hamill, R.L., Higgens, C.E., Boaz, H.E. and Gorman, M. (1969): The structure of beauvericin, a new depsipeptide antibiotic toxic to Artemia salina. Tetrahedron Lett., 10, 4255-4258.

Jow, G.M., Chou, C.J., Chen, B.F. and Tsai, J.H. (2004): Beauvericin induces cytotoxic effects in human acute lymphoblastic leukemia cells through cytochrome c release, caspase 3 activation: the causative role of calcium. Cancer Lett., 216, 165-173.

Karin, M. (1995): The regulation of AP-1 activity by mitogen-activated protein kinases. J. Biol. Chem., 270, 16483-16486.

Kunz, M., Ibrahim, S., Koczan, D., Thiesen, H.J., Köhler, H.J., Acker, T., Plate, K.H., Ludwig, S., Rapp, U.R., Bröcker, E.B., van Muijen, G.N., Flory, E. and Gross, G. (2001): Activation of c-Jun NH2-terminal kinase/stress-activated protein kinase (JNK/SAPK) is critical for hypoxia-induced apoptosis of human malignant melanoma. Cell Growth Differ., 12, 137-145.

Lin, H.I., Lee, Y.J., Chen, B.F., Tsai, M.C., Lu, J.L., Chou, C.J. and Jow, G.M. (2005): Involvement of Bcl-2 family, cytochrome c and caspase 3 in induction of apoptosis by beauvericin in human non-small cell lung cancer cells. Cancer Lett., 230, 248-259.

Lundberg, A.S. and Weinberg, R.A. (1999): Control of the cell cycle and apoptosis. Eur. J. Cancer, 35, 531-539.

Mosmann, T. (1983): Rapid colorimetric assay for cellular growth and survival: application to proliferation and cytotoxicity assays. J. Immunol. Methods, 65, 55-63.

Ojcius, D.M., Zychlinsky, A., Zheng, L.M. and Young, J.D. (1991): Ionophore-induced apoptosis: role of DNA fragmentation and calcium fluxes. Exp. Cell. Res., 197, 43-49.

Pietenpol, J.A. and Stewart, Z.A. (2002): Cell cycle checkpoint signaling: cell cycle arrest versus apoptosis. Toxicology, 181-182, 475-481.

Pucci, B., Kasten, M. and Giordano, A. (2000): Cell cycle and apop- 
Role of MAPK in beauvericin-induced cell apoptosis

tosis. Neoplasia, 2, 291-299.

Robinson, M.J. and Cobb, M.H. (1997): Mitogen-activated protein kinase pathways. Curr. Opin. Cell. Biol., 9, 180-186.

Roovers, K. and Assoian, R.K. (2000): Integrating the MAP kinase signal into the G1 phase cell cycle machinery. Bioessays, 22, 818-826.

Sandler, A.B., Nemunaitis, J., Denham, C., von Pawel, J., Comier, Y., Gatzemeier, U., Mattson, K., Manegold, C., Palmer, M.C., Gregor, A., Nguyen, B., Niyikiza, C. and Einhorn, L.H. (2000): Phase III trial of gemcitabine plus cisplatin versus cisplatin alone in patients with locally advanced or metastatic non-smallcell lung cancer. J. Clin. Oncol., 18, 122-130.

Schaeffer, H.J. and Weber, M.J. (1999): Mitogen-activated protein kinases: specific messages from ubiquitous messengers. Mol. Cell. Biol., 19, 2435-2444.
Stadheim, T.A., Saluta, G.R. and Kucera, G.L. (2000): Role of c-Jun $\mathrm{N}$-terminal kinase/p38 stress signaling in 1-beta-D-arabinofuranosylcytosine-induced apoptosis. Biochem. Pharmacol., 59, 407418.

Taylor, W.R. and Stark, G.R. (2001): Regulation of the G2/M transition by p53. Oncogene, 20, 1803-1815.

ten Bokkel Huinink, W.W., Bergman, G., Chemaissani, A., Dornoff, W., Drings, P., Kellokumpu-Lehtinen, P.L., Lippo, K., Mattson, K., von Pawel, J., Ricci, S., Sederholm, C., Stahel, R.A., Wagenius, G., Walree, N.V. and Manegold, C. (1999): Singleagent gemcitabine: an active and better tolerated alternative to standard cisplatin-based chemotherapy in locally advanced or metastatic non-small cell lung cancer. Lung Cancer, 26, 85-94.

Wada, T. and Penninger, J.M. (2004): Mitogen-activated protein kinases in apoptosis regulation. Oncogene, 23, 2838-2849. 